\title{
Les enjeux du développement de l'éducation thérapeutique
}

\section{Challenges in the development of therapeutic education}

\section{P.Y. Traynard $\cdot$ M. Varroud Vial}

Reçu le 20 septembre 2011 ; accepté le 23 septembre 2011

(C) Springer-Verlag France 2011

\section{Introduction}

Qui pouvait penser qu'il faudrait 45 ans après les premières publications de Miller et Goldstein [3] sur leurs expériences d'organisation de partages d'informations et de décisions avec des personnes socialement précaires, atteintes de diabète, pour que la loi HPST officialise, en France, le principe que « l'éducation thérapeutique fait partie intégrante de la prise en charge du patient et de son parcours de soins »?

Le chemin parcouru est long et on peut citer quelques repères. Si certains professionnels de santé ont toujours eu la « fibre pédagogique », c'est en 1975 que Gfeller et Assal ont publié leurs expérimentations de séances éducatives avec des groupes de patients [1]. Au début des années 1980, les formats hospitaliers se sont diversifiés avec des programmes d'éducation de jour, de semaine, de nuit, des stages dédiés. À partir des années 1990 naissent les premières applications dans le cadre des soins ambulatoires, portées par les réseaux de santé.

Parallèlement, les formations de soignants aux techniques d'éducation thérapeutique sont devenues accessibles à un plus grand nombre, avec une introduction assez récente dans les programmes de formation initiale.

Ces pratiques éducatives, qui répondent aux définitions de l'OMS en 1998 [4], ont été formalisées dans des recommandations et un guide par la Haute Autorité de santé (HAS) en 2007 [2] ; les compétences à acquérir par les patients, les notions d'équipe éducative, d'éducation initiale, de renforcement et de reprise ont ainsi été popularisées. Ces pratiques sont maintenant organisées et structurées dans des programmes soumis à l'autorisation des agences

P.Y. Traynard ( $\square)$

Réseau Paris Diabète, France

e-mail : traynard2@wanadoo.fr

M. Varroud Vial

Centre hospitalier Sud-Francilien, Corbeil-Essonnes, France régionales de santé, selon un cahier des charges basé sur les recommandations HAS.

L'éducation thérapeutique du patient (ETP) a été expérimentée au début dans la maladie diabétique, particulièrement le diabète de type 1 , dont le traitement dépend au plus haut point des compétences, des ajustements comportementaux et des adaptations psychologiques développés par le patient. Ses effets positifs sur la réduction de fréquence et de gravité des accidents liés à la maladie et à ses traitements étant démontrés, elle a été progressivement adoptée dans les autres spécialités médicales. Les sujets abordés dans ce dossier thématique témoignent d'une application de plus en plus large de ces approches fondamentalement humanistes de la médecine.

\section{Un changement fondamental dans les relations entre soignants et soignés}

Le développement des technologies médicales, l'allongement de la durée de vie avec son corollaire d'augmentation du nombre de personnes atteintes de maladies chroniques avec des traitements contraignants, quotidiens et permanents, auxquels les patients sont appelés à participer, la notion d'autodétermination en santé pouvant amener à considérer la santé comme un bien personnel sont autant de concepts qui sous-tendent l'émergence de la notion de compétences du patient. Cela change les rôles respectifs des soignants et des patients.

La référence universitaire traditionnelle, formatrice, et paradoxalement encore plus l'expérience hospitalière des étudiants renvoient à la séquence symptômes, diagnostic, acte dont la réussite mène à la guérison. À l'opposé, la maladie chronique, par son absence de finalité autre que la mort, exige du soignant un accompagnement à l'adaptation, et du patient un travail personnel d'arrangement avec la maladie. Ainsi, apprendre sur soi, pour soi, c'est acquérir suffisamment de compétences, d'autosémiologie, d'autosoins, de prises de décisions, d'adaptation à la maladie pour se mettre en 
sécurité au quotidien, reprendre sa place dans la vie avec les autres, conserver son estime de soi. Voici le temps d'un nouveau dialogue entre deux partenaires, l'un ayant un savoir acquis, universel, l'autre un savoir nourri d'expériences et d'essais. L'émergence de ces " nouveaux » savoirs, soidisant profanes, résulte aussi de la capacité des soignants à construire des programmes d'éducation qui visent réellement à permettre aux patients de s'approprier des procédures de soins souvent complexes. Elle débouche sur la notion de « parcours éducatifs ».

\section{De nouvelles compétences à acquérir par les soignants}

La démarche éducative, parce qu'elle est centrée sur les patients, renvoie les professionnels de santé à leurs propres compétences non seulement techniques mais aussi de coopération, pédagogiques et relationnelles. Un soignant, quel qu'il soit, peut-il en savoir assez pour répondre à toutes les questions et problèmes auxquels un patient peut être confronté dans sa vie quotidienne ? Cela pose la nécessité d'une entente pluriprofessionnelle et même parfois pluridisciplinaire. Recherche d'accords sur les contenus, référentiels communs, reconnaissance de savoirs et compétences spécifiques, intégration des soins et de l'éducation sont autant de nouveaux sujets de travaux et d'expérimentation.

Comprendre ce que le patient comprend, entendre dans quel projet de vie il peut s'inscrire maintenant que la maladie est là, établir ensemble quels écarts travailler entre ce qu'il fait et le souhaitable sont les bases d'un accord sur les perspectives d'ajustement de la vie avec la maladie. Ce temps de diagnostic éducatif, parce qu'il permet de s'entendre sur la pertinence et l'utilité de l'éducation, est le préalable à toute proposition au patient de participer à des activités d'éducation. Ces compétences pédagogiques et relationnelles nécessaires pour pratiquer l'ETP ne sont pas innées et ne s'inventent pas. Elles nécessitent un temps de formation personnelle, et le législateur ne s'y est pas trompé, qui demande une formation d'au moins 40 heures pour participer à l'ETP.

\section{Une révision des liens établis}

Les organisations hospitalières, particulières par l'unité de temps, celui de l'hospitalisation, de lieu, de service et d'équipe, relativement fixes, sont des références, représentant $75 \%$ des programmes autorisés... mais peu adaptés aux soins « de ville ». L'ETP ne trouvera sa cible réelle et son efficience que si elle déborde les limites de l'hôpital pour s'étendre davantage en ambulatoire. Et là, que l'on travaille seul, en lien avec d'autres professionnels de santé ou en équipe mono- ou pluriprofessionnelle, les pratiques d'ETP exigent tôt ou tard une réflexion sur comment intégrer l'éducation à sa pratique.

Ce sont les réseaux de santé qui, depuis plus de 20 ans, ont contribué à résoudre ces questions essentielles : comment coordonner les ressources et compétences professionnelles disponibles ? Où et comment trouver les ressources éducatives d'information, de documentation? Quelles sont les caractéristiques d'un programme d'éducation adapté au contexte ambulatoire ? Quels sont les lieux d'éducation en termes de proximité et d'accessibilité ? Comment déterminer les niveaux de besoins éducatifs des populations cibles ? Comment établir des liens entre les structures sanitaires, sociales et associations de patients?

\section{Trois enjeux majeurs}

Le premier est l'élaboration et l'expérimentation de programmes adaptés aux soins de ville. Ces programmes devront respecter certains critères : répondre à des problèmes fréquemment rencontrés en pratique de ville, faire une place à des séances d'éducation individuelles intégrées à la consultation ou au temps passé avec le patient, être réalisés de façon coopérative par les médecins, les pharmaciens, les infirmières, les diététiciennes..., être proposés et évalués par un ou plusieurs de ces acteurs.

Le deuxième est une harmonisation des programmes proposés par la ville (le plus souvent aujourd'hui au travers des réseaux de santé) et les établissements hospitaliers. Le but est de parvenir sur un même territoire à un maillage des offres éducatives, en s'accordant sur une complémentarité des programmes d'éducation selon l'expertise et le rôle de chacun et en mutualisant les moyens.

Le troisième est la mise en place de «pôles de ressources en ETP », apportant un appui aux professionnels de ville. Ceux-ci ne disposent pas encore facilement des ressources humaines, matérielles, pédagogiques, documentaires et financières pour répondre aux enjeux de l'ETP. Les «pôles de ressources en ETP », qui émergent dans plusieurs régions, visent à mettre en lien les organisations investies dans l'ETP, à participer à des actions de sensibilisation, à soutenir les mises en œuvre de pratiques éducatives, à faciliter le développement de programmes et la réponse aux appels d'offres censés les financer. Leurs actions devraient déboucher sur des partenariats institutionnels, hospitaliers, universitaires, et avec des instituts de formation en ETP.

Le développement et l'accessibilité des programmes d'ETP dépendent maintenant de choix clairement définis de politique de santé publique. 


\section{Références}

1. Gfeller R, Assal J-Ph (1980) The diabetic's subjective experience. In: Folia psychopractica. Hoffman-La Roche, Basel

2. Haute Autorité de santé, Inpes (2007) Structuration d'un programme d'éducation thérapeutique du patient dans le champ des maladies chroniques. Guide méthodologique. Saint-Denis La Plaine (France) : http://www.has-sante.fr/portail/jcms/c_601290/ structuration-dun-programme-deducation-therapeutique-du-patientdans-le-champ-des-maladies-chroniques

3. Miller L, Goldstein J (1972) More efficient care of diabetic patients in a county hospital setting. N Engl J Med 286:1388-91

4. OMS. Bureau régional pour l'Europe (1998) Éducation thérapeutique du patient. Programmes de formation continue pour professionnels de soins dans le domaine de la prévention des maladies chroniques. Copenhague (Danemark) : www.formatsante.org/ download/OMS.pdf

\section{包 Springer}

the language of science

\section{springerlink.com}

\section{SpringerLink}

\section{Access outstanding STM content with sophisticated search tools}

Books, journals, reference works, protocols \& more

Over 4 million documents on one integrated platform

More productive searches with Semantic Linking 